07

\title{
Томография в оптически осесимметричных средах
}

\author{
(C) А.Э. Пуро \\ Евроакадемия, \\ 10621 Таллинн, Эстония \\ e-mail: alfredpuro@gmail.com
}

Поступила в редакцию 30.08.2017г.

Предложено обобщение алгоритма Кормака обращения преобразования Радона в оптической среде с осесимметричным показателем преломления. Распространение лучей такое, что через две любые точки в круге проходит только один луч. Рассмотрена „параллельная схема“ томографии: цилиндрический объект просвечивается параллельным пучком света, лучи которого отклоняются внутри цилиндра, отсутствует рефракция на поверхности цилиндра. Алгоритм предполагает возможность затухания на луче, которое носит также осесимметричный характер. Такого типа отклонения лучей возникают в задачах томографии ГРАДАНОВ, световодов, плазмы.

DOI: $10.21883 /$ OS.2018.02.45536.205-17

\section{Введение}

В последние десятилетия в томографии тензорных полей (векторов и симметричных тензоров второго ранга) были получены существенные результаты: определены основные виды взаимодействия пробного излучения со средой, возможности реконструкции на основе лучевого интеграла той или иной компоненты тензора. Большинство результатов в этой области получены для случая прямолинейного распространения излучения. При распространении лучей по геодезическим линиям метрического пространства теория строится на основе конформного отображения. Предполагается, что между двумя любыми точками плоскости проходит только одна геодезическая кривая. Лучи в исследуемой плоскости конформно отражаются на плоскость так, что они становятся прямыми и соответственно задача приводится к виду, рассмотренному ранее. Так, в работе [1] представлен алгоритм обращения веерного преобразования Радона с поглощением при распространении лучей по геодезическим кривым (лучам) и представлены основные положения тензорной томографии. Ниже представлен алгоритм реконструкции преобразования Радона с „параллельной“ схемой сканирования при осесимметричном распределении показателя преломления оптической среды. Под параллельной схемой подразумевается, что лучи падают параллельно на исследуемый объект, внутри которого они распространяются по геодезическим кривым. Такого типа задачи встречаются при поляризационных исследованиях плазмы [2], оптической поляризационной томографии остаточных напряжений в ГРАДАНАХ [3] и световодах [4], в томографии акустических сред [5]. В большинстве этих задач предполагается слабое двулучепреломление. Распространение излучения описывается в рамках квазиизотропного приближения. Задача фактически разделяется на задачу распространения лучей в среде с изменяющимся показателем преломления и задачу трансформации по- ляризации на этих лучах. При распространении лучей в среде с осесимметричным распределением показателя преломления траектория лучей описывается формулой Бугера [6], что несколько упрощает построение алгоритма обращения преобразования Радона на луче. В том случае, когда поглощение на луче тоже аксиально симметрично, лучевой интеграл можно разложить по угловым гармоникам и его обращение построить по аналогии с алгоритмом Кормака $[7,8]$. Заметим, что с математической точки зрения алгоритм обращения справедлив и в том случае, когда поглощение является комплексной величиной. В частности, в задачах магнитофотоупругости [9] и исследованиях магнитного поля плазмы ТОКАМАКА [10] параметр, связанный с поглощением, носит мнимый характер и определяет фарадеевское вращение плоскости поляризации на луче. Здесь рассматривается именно такой случай. Во второй части работы представлен алгоритм определения потенциальной составляющей векторного поля по значениям лучевых интегралов с поперечным взаимодействием. Физически такому взаимодействию соответствуют измерения в интегральной фотоупругости и поляризационные измерения магнитного поля плазмы ТОКАМАКА.

\section{Алгоритм Кормака обращения преобразования Радона скалярной функции при рефракции луча}

В основу алгоритма решения задачи тензорной томографии с отклонением луча [1] положена формула обращения преобразования Радона скалярной функции. Изложение работы следует схеме двумерной томографии при „параллельном“ методе сканирования. Реконструкция осуществляется в круге единичного радиуса, причем через любые две точки внутри круга проходит только один луч. Значение функции одновременно 


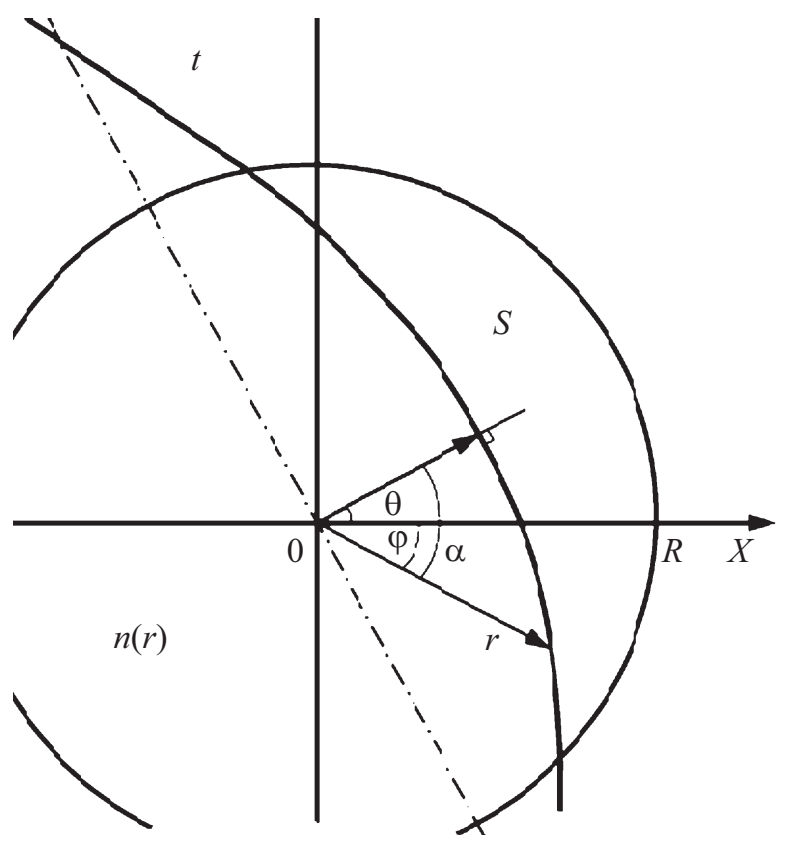

Рис. 1.

определяется в неподвижной системе координат $(x, y)$, в полярной системе координат $(r, \varphi)$ и в подвижной системе координат $(s, \theta)$, определяющей луч, на котором производится измерение (рис. 1). Напомним основные моменты построения траектории лучей в оптически симметричной среде. Будем исходить из формулы Бугера [6]:

$$
r n(r) \sin (v)=\operatorname{sn}(s),
$$

где $n(r)$ - показатель преломления, $v-$ угол между касательной к лучу и радиусом-вектором в точке касания, $s$ - кратчайшее расстояние от начала координат до кривой. Выражая

$$
\sin (v)=\frac{r(\alpha) d \alpha}{\sqrt{r^{2}(\alpha)(d \alpha)^{2}+(d r)^{2}}}
$$

через полярные координаты точки, получают траекторию луча:

$$
\alpha(r, s)=\int_{|s|}^{r} \frac{u(s) d \rho}{\rho \sqrt{u(\rho)^{2}-u(s)^{2}}} .
$$

Здесь $\alpha(r, s)= \pm(\varphi-\theta)$ - угол между радиусомвектором точки на кривой и осью $s, u(r)=r n(r)$. Отметим для справки, что действуя аналогично, можно получить уравнения кривых, ортогональных лучам (криволинейную ортогональную систему координат). В этом случае

$$
\alpha_{1}(r, s)=\int_{|s|}^{r} \frac{\sqrt{u(\rho)^{2}-u(s)^{2}} d \rho}{\rho u(s)} .
$$

Причем, если ось $s$ для лучей в формуле (1) направлена по оси $x$, то для кривых, ортогональных этой системе лучей, в формуле (2) ось $s$ направлена по оси $y$.
Предполагается, что $u(r)-$ монотонная функция, и существует взаимно однозначное соответствие между $u(r)$ и $r$ на отрезке $[0,1]$. Для лаконичности изложения в дальнейшем будем обозначать заглавными буквами аргумента переменную $u(r)=R(r)$. В новых обозначениях формула (1) примет следующий вид:

$$
\begin{aligned}
& \alpha[R(r), S(r)]=\int_{|s|}^{r} \frac{u(s) d \rho}{\rho \sqrt{u(\rho)^{2}-u(s)^{2}}}= \\
& \quad=\int_{|S|}^{R} \frac{S D(K) d K}{K \sqrt{K^{2}-S^{2}}}=A(R, S), \quad D(R)=\frac{d \ln r(R E)}{d \ln R} .
\end{aligned}
$$

Обращение рассматривается на круге единичного радиуса. Запишем преобразование Радона с мнимым поглощением в оптически симметричной среде в стандартной форме [1]:

$$
\hat{f}(s, \theta)=\int_{0}^{t} f(s \theta+x) \exp \left[i \int_{0}^{l} \mu(s \theta+x) d k\right] d l .
$$

Интегрирование ведется по лучу $t$ от точки входа в круг до точки выхода из области просвечивания, $\mu$ осесимметричная величина, характеризующая мнимое поглощение. Схема дальнейшего изложения следует аналогичной задаче для случая прямолинейного распространения лучей [8] и поэтому во многих местах объяснения даются в краткой форме. Преобразуем накопленное поглощение:

$$
\begin{aligned}
i M(R, S) & =i \int_{0}^{t} \mu(s \theta+x) d k=i \int_{|s|}^{r} \frac{\mu(k) u(k) d k}{\sqrt{[u(k)]^{2}-[u(s)]^{2}}} \\
& =i \int_{|S|}^{R} \frac{\mu(K) L(K) K d K}{\sqrt{K^{2}-S^{2}}},
\end{aligned}
$$

используя для этого выражение дифференциала дуги кривой $[2,3]$ :

$d l= \pm \frac{u(r) d r}{\sqrt{[u(r)]^{2}-[u(s)]^{2}}}= \pm \frac{L(R) R d R}{\sqrt{R^{2}-S^{2}}}, \quad L(R)=\frac{d r(R)}{d R}$.

Запишем лучевой интеграл в полярных координатах:

$$
\begin{gathered}
\hat{f}(S, \theta)=\int_{|S|}^{u(1)} \frac{f[K, \theta+A(K, S)] \exp [i M(K, S)] L(K) K d K}{\sqrt{K^{2}-S^{2}}} \\
+\int_{|S|}^{u(1)} \frac{f[K, \theta-A(K, S)] \exp [-i M(K, S)] L(K) K d K}{\sqrt{K^{2}-S^{2}}} .
\end{gathered}
$$


Разложим как саму функцию $f(R, \varphi)$, так и ее трансформанту в ряд по угловым гармоникам:

$$
\begin{aligned}
f(R, \varphi) & =\sum f_{m}(R) \exp (i m \varphi), \\
\hat{f}(S, \theta) & =\sum g_{m}(S) \exp (i m \theta) .
\end{aligned}
$$

Подставим их в предыдущее выражение. Сравнивая коэффициенты при одинаковых гармониках, получим интегральное уравнение

$$
\begin{aligned}
g_{m}(S, \mu) & =\frac{1}{2 \pi} \int_{0}^{2 \pi} \hat{f}(S, \theta) e^{-i m \theta} d \theta \\
& =2 \int_{|S|}^{u(1)} \frac{\cos [E(m, K, S, \mu)] F_{m}(K) K d K}{\sqrt{K^{2}-S^{2}}}
\end{aligned}
$$

(обобщенное уравнение Кормака) относительно $F_{m}(R)=f_{m}(R) L(R) . \quad$ Здесь $\quad E(m, K, S, \mu)=m A(K, S)+$ $+M(K, S)$.

Докажем, что решение уравнения можно записать в следующем виде:

$f_{m}(r) n(r)=\frac{-1}{2 \pi r}\left\{\frac{\partial}{\partial r}[\operatorname{Cr} 1(g, R, \mu)]+\frac{m}{r}[\operatorname{Sr} 1(g, R, \mu)]\right\}$.

Здесь

$$
\begin{gathered}
C r 1(g, R, \mu)=C r(g, R, \mu)+C r(g, R,-\mu), \\
\operatorname{Cr}(g, R, \mu)=\int_{R}^{u(1)} \frac{\operatorname{ch}[G(m, S, R, \mu)] g_{m}(S, \mu) S d S}{\sqrt{S^{2}-R^{2}}}, \\
\operatorname{Sr} 1(g, R, \mu)=\operatorname{Sr}(g, R, \mu)+\operatorname{Sr}(g, R,-\mu), \\
\operatorname{Sr}(g, R, \mu)=\int_{R}^{u(1)} \operatorname{sh}[G(m, S, R, \mu)] g_{m}(f, S, \mu) d S, \\
G(m, S, R, \mu)=m B(S, R)+N(S, R), \\
B(S, R)=\int_{R}^{|S|} \frac{S D(K) d K}{K \sqrt{S^{2}-K^{2}}}, \\
N(S, R, \mu)=\int_{R}^{|S|} \frac{\mu(K) L(K) K d K}{\sqrt{S^{2}-K^{2}} .}
\end{gathered}
$$

Для этого подставим в решение интегрального уравнения (5) трансформанту (4):

$$
\begin{aligned}
& f_{m}(r) n(r)=\frac{-1}{2 \pi r}\left\{\frac { \partial } { \partial r } \left[\int_{R}^{u(1)} \frac{\operatorname{ch}[G(m, S, R, \mu)] S}{\sqrt{S^{2}-R^{2}}}\right.\right. \\
& \left.\times \int_{|S|}^{u(1)} \frac{\cos [E(m, K, S, \mu)] F_{m}(K) K d K}{\sqrt{K^{2}-S^{2}}} d S+\ldots\right] \\
& +\frac{m}{r}\left[\int_{R}^{u(1)} \operatorname{sh}[G(m, S, R, \mu)]\right. \\
& \left.\left.\times \int_{|S|}^{u(1)} \frac{\cos [E(m, K, S, \mu)] F_{m}(K) K d K}{\sqrt{K^{2}-S^{2}}} d S+\ldots\right]\right\} .
\end{aligned}
$$

В скобках многоточием обозначены интегралы, содержащие $\mu$ со знаком минус. Следуя схеме работы [8], преобразуем интегралы в квадратных скобках. Первоначально преобразуем интегралы в первой квадратной скобке, меняя в них порядок интегрирования:

$\left[\int_{R}^{u(1)} \frac{\operatorname{ch}[G(m, S, R, \mu)] S}{\sqrt{S^{2}-R^{2}}}\right.$

$$
\begin{aligned}
& \left.\times \int_{|S|}^{u(1)} \frac{\cos [E(m, K, S, \mu)] F_{m}(K) K d K}{\sqrt{K^{2}-S^{2}}} d S+\ldots\right]=\int_{R}^{u(1)} F_{m}(K) K \\
& \times\left[\int_{R}^{K} \frac{\operatorname{ch}[G(m, S, R, \mu)] \cos [E(m, K, S, \mu)]}{\sqrt{K^{2}-S^{2}} \sqrt{S^{2}-R^{2}}} S d S+\ldots\right] d K .
\end{aligned}
$$

Применим теорию комплексной переменной для доказательства тождества

$$
\begin{aligned}
G 1 & =\int_{R} \frac{+\operatorname{ch}[G(m, S, R, \mu)] \cos [E(m, K, S, \mu)]+}{\sqrt{K^{2}-S^{2}} \sqrt{S^{2}-R^{2}}} \\
& =\frac{\pi}{2}\left[\left(\frac{k(K)}{r(K)}\right)^{m}+\left(\frac{r(R)}{k(K)}\right)^{m}\right] .
\end{aligned}
$$

Используя тригонометрическое тождество $\cos (A) \operatorname{ch}(B)=$ $=\operatorname{Re}[\operatorname{ch}(B-i A)]$, где $A, B-$ действительные числа, запишем интеграл $G 1$ как действительную часть комплексного интеграла:

$$
\begin{aligned}
& \operatorname{ch}[G(m, S, R, \mu)-i E(m, K, S, \mu)]+ \\
& G 1=\operatorname{Re} \int_{R}^{K} \frac{+\operatorname{ch}[G(m, S, R,-\mu)-i E(m, K, S,-\mu)]}{\sqrt{K^{2}-S^{2}} \sqrt{S^{2}-R^{2}}} S d S .
\end{aligned}
$$




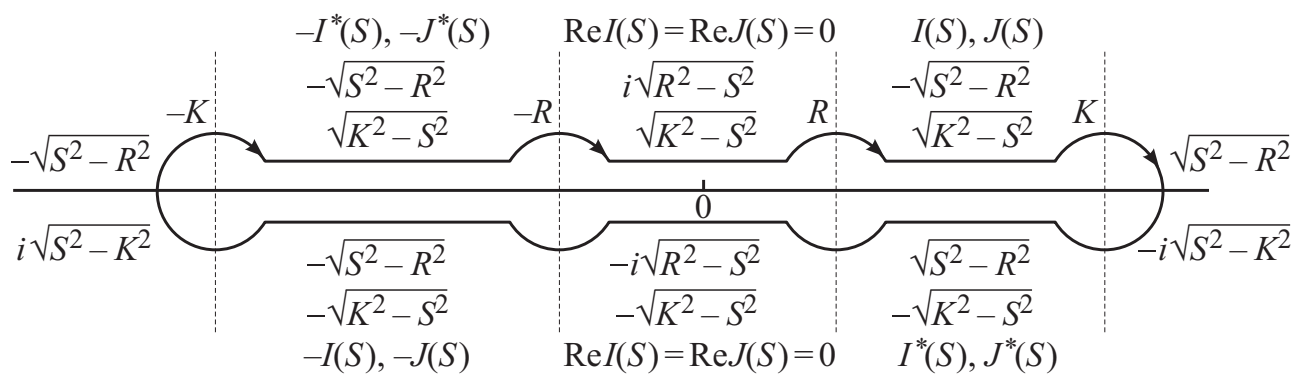

Рис. 2.

Здесь

$$
\begin{aligned}
G+i E= & m\left[\int_{R}^{|S|} \frac{S D(T) d T}{T \sqrt{S^{2}-T^{2}}}-i \int_{|S|}^{K} \frac{S D(T) d T}{T \sqrt{T^{2}-S^{2}}}\right] \\
& +\left[\int_{R}^{|S|} \frac{\mu(T) L(T) T d T}{\sqrt{S^{2}-T^{2}}}-i \int_{|S|}^{K} \frac{\mu(T) L(T) T d T}{\sqrt{T^{2}-S^{2}}}\right] \\
& =m\left[\int_{R}^{K} \frac{S D(T) d T}{T \sqrt{S^{2}-T^{2}}}\right]+\left[\int_{R}^{K} \frac{\mu(T) L(T) T d T}{\sqrt{S^{2}-T^{2}}}\right] \\
& =m I(K, R)+J(K, R, \mu) .
\end{aligned}
$$

Вычисление интеграла $G 1$ заменим вычислением контурного интеграла:

$$
G 1=\frac{1}{4} \operatorname{Re} \oint \frac{2 \operatorname{ch}[m I(K, R)] \operatorname{ch}[J(K, R, \mu)]}{\sqrt{K^{2}-S^{2}} \sqrt{S^{2}-R^{2}}} S d S .
$$

Интегрирование производится по контуру по часовой стрелке с обходом полюсов как указано на рис. 2. Звездочкой на рисунке отмечена операция комплексного сопряжения и соответствующие преобразования интегралов на контуре. Нетрудно заметить, что значения контурного интеграла $G 1$ на участках $1,2,4,5$ совпадают, а на участках 3 и 6 равны нулю. Благодаря разрезам подынтегральная функция аналитическая вне контура. Запишем разложение аргумента гиперболического косинуса в ряд Лорана по $S$ при $|S|>K$ :

$$
I(K, R)=m\left[\int_{R}^{K} \frac{S D(T) d T}{T \sqrt{S^{2}-T^{2}}}\right]=\sum_{k=0}^{\infty} a_{k} S^{-k},
$$

где

$$
\begin{aligned}
a_{0} & =m \int_{R}^{K} \frac{D(T) d T}{T}=m \int_{R}^{K} \frac{d \ln [\rho(T)] d T}{T d \ln (T)} \\
& =m \int_{r(R)}^{r(K)} \frac{d \rho}{\rho}=\ln \left[\frac{k(K)}{r(R)}\right]^{m} .
\end{aligned}
$$

Разложение функции $J(K, R, \mu)$ в ряд Лорана содержит только отрицательные степени переменной $S$. Таким образом, интегрируя по окружности радиуса, большего $K$, и используя теорему Коши, получаем необходимое тождество:

$$
\begin{aligned}
G 1 & =\frac{1}{4} \oint \frac{2 \operatorname{ch}[m \operatorname{In}[k(K) / r(R)]]}{-i S} d S \\
& =\frac{\pi}{2}\left[\left(\frac{k(K)}{r(R)}\right)^{m}+\left(\frac{r(R)}{k(K)}\right)^{m}\right] .
\end{aligned}
$$

Действуя аналогичным методом, преобразуем интеграл во второй квадратной скобке выражения (6):

$$
\begin{aligned}
& {\left[\int_{R}^{u(1)} \operatorname{sh}[G(m, S, R, \mu)]\right.} \\
& \left.\times \int_{|S|}^{u(1)} \frac{\cos [E(m, K, S, \mu)] F_{m}(K) K d K}{\sqrt{K^{2}-S^{2}}} d S+\ldots\right] \int_{R}^{u(1)} F_{m}(K) K \\
& \times\left[\int_{R}^{K} \frac{\operatorname{sh}[G(m, K, S, \mu)] \cos [E(m, K, S, \mu)]}{\sqrt{K^{2}-S^{2}}} d S+\ldots\right] d K .
\end{aligned}
$$

Повторяя предыдущие рассуждения, выразим интеграл в квадратной скобке через контурный интеграл

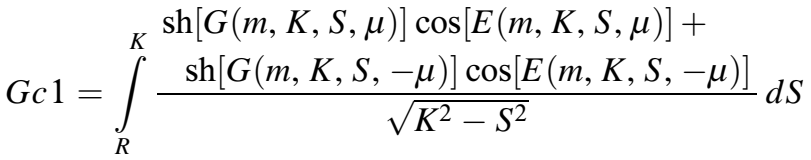

$$
\begin{aligned}
& \operatorname{sh}[G(m, K, S, \mu)-i E(m, K, S, \mu)]+ \\
& =\frac{1}{4} \operatorname{Re} \oint \frac{\operatorname{sh}[G(m, K, S,-\mu)-i E(m, K, S,-\mu)]}{\sqrt{K^{2}-S^{2}}} d S .
\end{aligned}
$$

Учитывая, что контурный интеграл вне круга радиуса $|S|>K$ аналитическая функция, и используя теорему Коши, получаем

$$
\begin{aligned}
G c 1 & =\frac{1}{4} \operatorname{Re} \oint \frac{2 \operatorname{sh}[m I(K, R)] \operatorname{ch}[J(K, R, \mu)]}{\sqrt{K^{2}-S^{2}}} d S \\
& =\frac{\pi}{2}\left[\left(\frac{k(K)}{r(R)}\right)^{m}-\left(\frac{r(R)}{k(K)}\right)^{m}\right] .
\end{aligned}
$$


Для завершения доказательства подставим значения интегралов (8), (10) в проверяемое решение (5):

$$
\begin{aligned}
& f_{m}(r) n(r)= \\
& =\frac{-1}{2 r}\left\{\frac{\partial}{\partial r}\left[\int_{R}^{u(1)}\left[\left(\frac{k(K)}{r(R)}\right)^{m}+\left(\frac{r(R)}{k(K)}\right)^{m}\right] F_{m}(K) K d K\right]\right. \\
& \left.+\frac{m}{r}\left[\int_{R}^{u(1)}\left[\left(\frac{k(K)}{r(R)}\right)^{m}-\left(\frac{r(R)}{k(K)}\right)^{m}\right] F_{m}(K) K d K\right]\right\},
\end{aligned}
$$

и вычислим производную. В результате получим тождество. Используя элементарные преобразования, можно записать решение (5) в более привычной форме [11]:

$$
\begin{gathered}
f_{m}(r)=\frac{d[u(r)]}{2 \pi d r}\left\{\operatorname{Cr}\left(g^{\prime}, R, \mu\right)+C r\left(g^{\prime}, R,-\mu\right)\right. \\
-[S r 2(R, \mu)+S r 2(R,-\mu)]\}, \\
C r\left(g^{\prime}, R, \mu\right)=\int_{R}^{u(1)} \frac{\operatorname{ch}[G(m, S, R, \mu)]}{\sqrt{S^{2}-R^{2}}} g_{m}^{\prime}(S, \mu) d S, \\
\operatorname{Sr} 2(R, \mu)=\int_{R}^{u(1)} \frac{\operatorname{sh}[G(m, S, R, \mu)]}{\sqrt{S^{2}-R^{2}}} g_{m}(S, \mu) \\
\quad\left[m \int_{R}^{S} \frac{D^{\prime}(K) d K}{\sqrt{S^{2}-K^{2}}}-\int_{R}^{S} \frac{S[\mu(K) L(K) K]^{\prime} d K}{\sqrt{S^{2}-K^{2}}}\right] d S .
\end{gathered}
$$

Здесь штрих обозначает производную. В представлении (12) явно выражены слагаемые, зависящие от кривизны лучей и от ослабления излучения. При прямолинейном распространении лучей значение показателя преломления $n$ - постоянно, значение $D(R)=1 / n-$ постоянно и интеграл $I(K, R)$ вычисляется в явном виде. Поэтому представленные решения незначительно отличаются от соответствующих выражений при прямолинейном распространении лучей $[12,13]$. Как указывалось, почти все многочисленные свойства преобразования Радона без рефракции автоматически переносятся на преобразования с рефракцией. Здесь в качестве иллюстрации этого факта приведем вывод алгоритма реконструкции потенциальной составляющей векторного поля по томографическим измерениям лучевых интегралов с поперечным взаимодействием.

\section{Алгоритм реконструкции векторного поля при поперечном взаимодействии}

Во многих прикладных задачах тензорной томографии возникает проблема реконструкции векторного поля на основе томографических измерений. Выделяют отдельно случаи с поперечным и продольным взаимодействием пробного излучения (поляризованного света) с векторным полем. В случае прямолинейного распространения лучей алгоритм реконструкции хорошо известен: при поперечном взаимодействии восстанавливается потенциальная составляющая векторного поля. При ослаблении излучения может быть восстановлена и соленоидальная часть поля. При продольном взаимодействии восстанавливается соленоидальная часть. При ослаблении излучения может быть восстановлена потенциальная часть векторного поля. В случае рефракции света все вышеперечисленные свойства томографии векторного поля сохраняются. Ниже ограничимся выводом алгоритма только потенциальной составляющей векторного поля при поперечном взаимодействии.

Представим векторное поле в полярной системе координат, разложив его на потенциальную и соленоидальную части:

$$
W=\left(\frac{\partial \tau}{\partial r}+\frac{\partial N}{r \partial \varphi}\right) e_{r}+\left(\frac{\partial N}{\partial r}-\frac{\partial \tau}{r \partial \varphi}\right) e_{\varphi} .
$$

Здесь $\tau$ и $N$ - потенциалы потенциальной и соленоидальной частей вектора $\mathbf{W}, \mathbf{e}_{r}$ и $\mathbf{e}_{\varphi}-$ соответствующие единичные векторы. Для краткости изложения предположим, что потенциалы удовлетворяют граничным условиям $\tau(1)=0, N(1)=0$. При поперечном взаимодействии измеряется лучевой интеграл от ортогональной составляющей вектора на луч [9]:

$$
W_{n}=\left[\left(\frac{\partial \tau}{\partial r}+\frac{\partial N}{r \partial \varphi}\right) \sin v+\left(\frac{\partial N}{\partial r}-\frac{\partial \tau}{r \partial \varphi}\right) \cos v\right] .
$$

Здесь

$$
\sin v=\frac{u(s)}{u(r)}, \quad \cos v=\mp \frac{\sqrt{u^{2}(r)-u^{2}(s)}}{u(r)},
$$

знак минус соответствует левой половине луча относительно оси $s$, плюс - правой. Подставляя (13) в (3), получим коэффициент Фурье трансформанты Радона, выраженный через угловые гармоники $\tau_{m}, N_{m}$. Выпишем сначала часть, содержащую потенциал $\tau$ :

$$
\begin{gathered}
w_{m}(\tau, S, \mu)=2 \int_{|S|}^{u(1)} \frac{\cos [E(m, K, S)] S}{\sqrt{K^{2}-S^{2}}} \frac{\partial \tau_{m}(K)}{\partial K} \\
-\frac{m \sin [E(m, K, S)]}{k(K)} \frac{d k(K)}{d K} \tau_{m}(K) d K .
\end{gathered}
$$

Покажем, что реконструкция потенциала $\tau$ осуществляется оператором

$$
\tau_{m}(R)=-\frac{1}{\pi} \operatorname{Cr} 1\left(w_{m}(\tau, S, \mu), R, \mu\right) .
$$

Заметим, что формула (15) состоит из двух слагаемых, соответствующих трансформанте Радона (14). Обращение первого слагаемого аналогично обращению 
интеграла (8), поэтому займемся обращением второго слагаемого:

$$
\begin{aligned}
& \frac{2}{\pi}\left[\int_{R}^{u(1)} \frac{\operatorname{ch}[G(m, S, R, \mu)]}{\sqrt{S^{2}-R^{2}}}\right. \\
& \left.\times \int_{|S|}^{u(1)} \frac{m \sin [E(m, K, S)]}{k(K)} \frac{d k(K)}{d K} \tau_{m}(K) d K d S+\ldots\right]= \\
& =\frac{2 m}{\pi} \int_{R}^{u(1)} \frac{d k(K)}{d K} \frac{\tau_{m}(K)}{k(K)} \\
& \times\left[\int_{R}^{K} \frac{\operatorname{ch}[G(m, S, R, \mu)] \sin [E(m, K, S, \mu)]}{\sqrt{S^{2}-R^{2}}} d S+\ldots\right] d K .
\end{aligned}
$$

Многоточием отмечены аналогичные слагаемые, содержащие $\mu$ со знаком минус. Следуя вышеизложенной методике, преобразуем выражение в квадратной скобке в контурный интеграл. Используя элементарные преобразования, запишем его в следующем виде:

$$
\begin{gathered}
\left.\left.G 3=\int_{R} \frac{\operatorname{ch}[G(m, S, R, \mu)] \sin [E(m, K, S, \mu)]+}{\sqrt{S^{2}-R^{2}}} d m, S, R,-\mu\right)\right] \sin [E(m, K, S,-\mu)] \\
\sqrt{K} \\
\left.\left.=-\operatorname{Im} \int_{R}^{K} \frac{\operatorname{sh}[G(m, S, R, \mu)-i E(m, K, S, \mu)]+}{\sqrt{S^{2}-R^{2}}} d S . S, R,-\mu\right)-i E(m, K, S,-\mu)\right]
\end{gathered}
$$

Далее преобразуем его в контурный интеграл и вычислим его значение:

$$
\begin{aligned}
G 3 & =-\frac{1}{4} \operatorname{Im} \oint \frac{2 \operatorname{sh}[m I(K, R)] \operatorname{ch}[J(K, R, \mu)]}{\sqrt{S^{2}-R^{2}}} d S \\
& =-\frac{\pi}{2}\left[\left(\frac{k(K)}{r(R)}\right)^{m}-\left(\frac{r(R)}{k(K)}\right)^{m}\right] .
\end{aligned}
$$

Здесь интегрирование происходит по контуру против часовой стрелки. При вычислении интеграла учтены свойства подынтегрального выражения, отмеченные на рис. 2. Подставляя полученные результаты в (15),

$$
\begin{gathered}
\tau_{m}(R)=\frac{-1}{2}\left\{\left[\int_{R}^{u(1)}\left[\left(\frac{k(K)}{r(R)}\right)^{m}+\left(\frac{r(R)}{k(K)}\right)^{m}\right] \frac{d \tau_{m}(K)}{d K} d K\right]\right. \\
\left.+m\left[\int_{R}^{u(1)}\left[\left(\frac{k(K)}{r(R)}\right)^{m}-\left(\frac{r(R)}{k(K)}\right)^{m}\right] \frac{d k(K)}{d K} \frac{\tau_{m}(K)}{k} d K\right]\right\},
\end{gathered}
$$

Рассмотрим действие обратного оператора (12) на часть, содержащую потенциал $N$ :

$$
\begin{aligned}
& w_{m}(N, S, \mu)=2 i \int_{|S|}^{u(1)} \sin [E(m, K, S)] \frac{\partial N_{m}(K)}{\partial K} d K \\
& +2 i m \int_{|S|}^{u(1)} \frac{\cos [E(m, K, S)] S}{\sqrt{K^{2}-S^{2}}} \frac{d k(K)}{d K} \frac{N_{m}(K)}{k(K)} d K .
\end{aligned}
$$

Покажем, что

$$
-\frac{1}{\pi} \operatorname{Cr} 1\left(w_{m}(N, S, \mu), R, \mu\right)=0 .
$$

Подставляя выражение (18) в (19) и меняя порядок интегрирования, приходим к ранее рассмотренным контурным интегралам (16) и (10). В результате элементарных преобразований выражение (19) преобразуется к легко проверяемому тождеству

$$
\begin{aligned}
& \left\{\left[\int_{R}^{u(1)}\left[\left(\frac{k(K)}{r(R)}\right)^{m}-\left(\frac{r(R)}{k(K)}\right)^{m}\right] \frac{d N_{m}(K)}{d K} d K\right]\right. \\
& \left.+m\left[\int_{R}^{u(1)}\left[\left(\frac{k(K)}{r(R)}\right)^{m}+\left(\frac{r(R)}{k(K)}\right)^{m}\right] \frac{d k(K)}{d K} \frac{N_{m}(K)}{k} d K\right]\right\}=0 .
\end{aligned}
$$

Здесь при интегрировании по частям первого интеграла необходимо учитывать, что на границе круга по условию потенциал $N$ равен нулю. Таким образом, было показано, что оператор (15) восстанавливает потенциальную часть векторного поля по значениям лучевого интеграла с поперечным взаимодействием. Аналогичное свойство имеем при прямолинейном распространении лучей в среде без рефракции.

В заключение еще раз отметим, что цель работы показать, как изменяются алгоритмы реконструкции преобразования Радона в условиях рефракции лучей. Большинство рассмотренных задач в интегральной фотоупругости с незначительными изменениями могут быть решены в случае рефракции лучей с учетом приведенных примеров. Сами алгоритмы являются основой для разработки устойчивых численных методов обращения. Этому вопросу посвящена обширная литература, применительно к осесимметричному поглощению этот вопрос частично освещен в работе [8].

\section{Список литературы}

[1] Monard F. // Inv. Probl. Imag. 2016. V. 10. N 2. P. 433.

[2] Illingworth R. // Opt. Commun. 1977. V. 23. N 1. P. 109.

[3] Каров Д.Д. Автореф. канд. дис. СПб. 2012. Электронный ресурс. Режим доступа: http:/fizmathim.com/ $/ \mathrm{read} / 369384 / \mathrm{a}$ ?\#? page $=16$

получаем легко проверяемое тождество. 
[4] Pagnotta L., Poggialini A. // Soc. Exp. Mech. 2003. V. 43. N 1. P. 69.

[5] Sharafutdinov V., Wang J.-N. // Inv. Probl. 2012. V. 28. N 6. doi 10.1088/0266-5611/28/6/065017

[6] Born M., Wolf E. Principal of optics. Oxford: Pergamon Press, 1968. 808 р. [Пер. М. Борна, Э. Вольфа. Основы оптики. М.: Наука, 1973. 720 c.]

[7] Cormack A.M. // J. Appl. Phys. 1963. V. 34. N 9. P. 2722.

[8] Puro A., Garin A. // Inv. Probl. 2013. V. 29. N 6. P. 065004. doi 10.1088/0266-5611/29/6/065004

[9] Puro A. // Inv. Probl. 1998. V. 14. P. 1315.

[10] Пуро А. // ОПт. и спектр. 2014. Т. 116. № 1. С. 135; Puro A. // Opt. Spectrosc. 2014. V. 116. N 1. P. 122.

[11] Natterer $F$. The Mathematics of Computerized Tomography. Stuttgart: Teubner, 1986. 225 p. Пер. Ф. Наттерера. Математические аспекты компьютерной томографии. М.: Мир, 1990. 279 c. doi 10.1007/978-3-663-01409-6

[12] Riguad G., Lakhal A. // Inv. Probl. 2015. V. 31. P. 025007. doi 10.1088/0266-5611/31/2/025007

[13] Пуро А. // Опт. и спектр. 2011. Т. 110. № 6. С. 1002; Puro A. // Opt. Spectrosc. 2011. V. 110. N 6. P. 947. 